\title{
Gene-Eden-VIR Decreased Physical and Mental Fatigue in a Post Marketing Clinical Study That Followed FDA Guidelines; Results Support Microcompetition Theory
}

\author{
Hanan Polansky*, Edan Itzkovitz \\ Center for the Biology of Chronic Disease (CBCD), Rochester, USA \\ Email: ${ }^{*}$ ppolansky@cbcd.net
}

Received 15 January 2014; revised 16 February 2014; accepted 6 March 2014

Copyright (C) 2014 by authors and Scientific Research Publishing Inc.

This work is licensed under the Creative Commons Attribution International License (CC BY). http://creativecommons.org/licenses/by/4.0/

(c) (i) Open Access

\section{Abstract}

Objective: The Microcompetition with Foreign DNA theory, proposed by Hanan Polansky in 2003, describes how latent viruses can cause chronic conditions, including fatigue. The Gene-Eden-VIR formula was designed to target latent viruses. Therefore, the theory predicts that treatment with Gene-Eden-VIR will decrease fatigue in individuals infected with a latent virus. The objective of this study was to test this prediction. Framework: A post marketing clinical study that followed FDA guidelines. Treatment: Gene-Eden-VIR, a dietary supplement. A capsule of Gene-Eden-VIR includes $100 \mathrm{mg}$ of quercetin, $150 \mathrm{mg}$ of green tea extract, $50 \mathrm{mg}$ of cinnamon extract, $25 \mathrm{mg}$ of licorice extract, and $100 \mathrm{mcg}$ of selenium. The treatment included 1, 2, 3, or 4 capsules per day, and lasted 2 to 54 weeks. Population: 100 individuals infected with a latent virus, including the Human Papillomavirus (HPV), Epstein Barr Virus (EBV), Herpes Simplex Virus (HSV), Human Cytomegalovirus (HCMV), and Hepatitis C Virus (HCV). Ages ranged from 20 to 66. All participants reported a feeling of fatigue at the start of the study. Specifically, 98, 90, and 79 participants reported a feeling of general, physical, and mental fatigue, respectively. Results: Following treatment with GeneEden-VIR, $73.47 \%, 62.22 \%$, and $47.36 \%$ reported a decrease in their feeling of general, physical, and mental fatigue, respectively. The participants also reported a statistically significant decrease in every aspect of fatigue tested in the study. The results also showed a duration effect, that is, those treated for $\mathbf{2}$ months or more reported a larger decrease in their feeling of fatigue (general, $p=0.03, n=65$; physical and mental, $p=0.05, n=70$ ). The results showed no interviewer bias, and no selection bias. In addition, the results showed therapeutic consistency under varying manufacturing conditions. The participants reported no side effects after taking Gene-Eden-VIR. Conclusions: This post marketing clinical study showed that treatment with Gene-Eden-VIR safely decreased the feeling of general, physical, and mental fatigue in individuals infected with a latent vi-

\footnotetext{
${ }^{*}$ Corresponding author.
} 
rus. Since most individuals are infected with a latent virus, health care practitioners should recommend Gene-Eden-VIR as a first line treatment for fatigue. The results of this post marketing clinical study support the Microcompetition with Foreign DNA theory.

\section{Keywords}

Gene-Eden-VIR; Microcompetition; Fatigue; Mental Fatigue; Physical Fatigue; Latent; Virus; Human Papillomavirus; HPV; Epstein Barr Virus; EBV; Herpes Simplex Virus; HSV; Cytomegalovirus; CMV; Hepatitis C Virus; HCV

\section{Introduction}

This paper reports the results of a post marketing clinical study that tested the effect of Gene-Eden-VIR, an antiviral natural formulation, on fatigue. Specifically, the clinical study tested the effect of Gene-Eden-VIR on the feeling of general, physical, and mental fatigue. The population included individuals infected with a latent virus, including the Human Papillomavirus (HPV), Epstein Barr Virus (EBV), Herpes Simplex Virus (HSV), Human Cytomegalovirus (HCMV), and Hepatitis C Virus (HCV).

Studies have shown that one-third of the general population reports a feeling of fatigue, with $5 \%$ reporting a feeling of short-term fatigue ( $<6$ months), and $31 \%$ reporting a feeling of long term or chronic fatigue ( $>6$ months) [1]. In the US alone, the feeling of fatigue is responsible for 10 - 15 million visits to the family doctor every year [2].

Studies associate the feeling of fatigue with a sense of tiredness, lack of energy, a feeling of exhaustion, and impaired physical and/or cognitive performance [3].

Various diseases (e.g., anemia, multiple sclerosis, CFS), psychiatric disorders, and drugs (e.g., antihypertensives, $\beta$-adrenergic blockers, lipid-lowering agents) can cause a feeling of fatigue. One of the most important causes of a feeling of fatigue is an infection, specifically, with a latent virus, such as the Epstein-Barr virus (EBV), human herpesvirus 6 (HHV-6), human herpesvirus 7 (HHV-7), herpes simplex virus (HSV), human immunodeficiency virus (HIV), hepatitis viruses, parvovirus B19, and influenza virus [4]-[7].

Most scientists and physicians believe that latent viruses cause no pathologies or symptoms. The FDA even states on its website: "Some viruses, however, can enter a state known as latency in which the virus is not being replicated. In the latent state, the virus does not cause disease” [8]. As a result, current standard medical practices don't target latent viruses.

In contrast to this belief, the Microcompetition with Foreign DNA theory [9], proposed by Hanan Polansky in 2003, describes how viruses, during their latent phase, can cause a chronic disease.

The theory was presented in a book entitled "Microcompetition with Foreign DNA and the Origin of Chronic Disease". The theory describes the effect of Foreign DNA fragments that include the cis-regulatory element, called an N-box, on transcription of cellular genes.

Consider foreign N-boxes that entered the body naturally, through, for instance, an infection or digestion, or artificially, through, for instance, an injection of some treatment. The foreign N-boxes attract the transcription complex GABP-p300. Since the complex is a scarce genetic resource, the foreign N-boxes decrease the availability of this complex to cellular genes. As a result, the genes that are transactivated by the GABP·p300 complex produce fewer proteins, and the genes that are suppressed by this complex produce more proteins. The abnormal levels of these cellular proteins cause a disease.

It is interesting that many common viruses that establish a latent infection have strong N-boxes in their promoter/enhancer. They include the Epstein-Barr virus (EBV), Cytomegalovirus (CMV), Herpes Simplex virus (HSV), Varicella Zoster virus (VZV), Hepatitis B Virus (HBV), Hepatitis C Virus (HCV), and the Human Papillomavirus (HPV). In fact, the CMV has the strongest promoter/enhancer known to science. Liu et al. showed that the CMV promoter/enhancer, which includes the N-box, is more than 150-fold stronger than the promoter of the platelet-derived growth factor-b chain (PDGF-b) gene [10].

During latency, an infected cell harbors about 10 copies of the CMV [11]. Multiplying the two numbers, about 10 copies of CMV per cell during latency, and 150-fold stronger promoter/enhancer, suggests that a latent infection with CMV has a similar effect on the PDGF-b promoter, and hence, its transcription, as the introduc- 
tion of $10 \times 150$, or 1500 copies of additional PDGF-b genes into the cell. Since, PDGF-b is susceptible to microcompetition with CMV [12], the Microcompetition theory predicts that a latent infection with CMV will cause a decrease in PDGF-b transcription, a decrease in the concentration of the PDGF-b protein, and disease.

Studies have shown that GABP is involved in the mitochondria electron transport chain. For instance, several studies have shown that GABP transactivates the expression of the gene encoding the cytochrome oxidase subunit IV (COXIV), a terminal enzyme of the mitochondrial respiratory chain [13] [14]. GABP also transactivates the COX subunit $\mathrm{Vb}$ gene, another enzyme of the mitochondrial respiratory chain. Several studies indicated a direct role for GABP in the expression of all 10 nucleus-encoded cytochrome oxidase subunits. For instance, Ongwijitwat et al. associated GABP in vivo with multiple COX subunit promoters by chromatin immunoprecipitation [15]. Ongwijitwat et al. also showed that expression of a dominant negative GABP allele decreased COX expression, and that an siRNA directed against GABP $\alpha$ decreased expression of all 10 COX subunits [16]. In addition, studies identified functional N-Boxes in a number of other genes related to the respiratory chain. These include genes for Tfam [17] [18] and TFB1M and TFB2M [19] [20], and three human succinate dehydrogenase (complex II) subunit genes [21]-[23].

Other studies have also shown that GABP transactivates the expression of the neuromuscular proteins utrophin [24], acetylcholine esterase (AChE) [25], and acetylcholine receptor subunits delta (AChRd and epsilon (AChRe) through their N-box promoter/enhancer cis-regulatory element [26].

Tinsely et al. showed that a small molecule that upregulates utrophin expression decreased the dystrophic symptoms in the mdx mouse [27]. Specifically, the study showed that "Treatment results in reduced pathology, better muscle physiology leading to an increase in overall strength, and an ability to resist fatigue after forced exercise".

Faber et al. showed that mutations in AChRe subunit gene are underlying the Congenital Myasthenic Syndromes (CMS) in nine patients of Dutch origin [28]. CMS are neuromuscular diseases that show abnormal muscle weakness and fatigue. It is interesting that CMS patients also show a marked deficiency in utrophin at the neuromuscular junctions [29].

Acetylcholinesterase inhibitors ("anticholinesterase") cause muscle spasms and twitches and decrease muscle strength. Payne et al. showed that administration of neostigmine, an AChE inhibitor, to an anaesthetized man can result in muscle weakness [30]. Caldwell calls it paradoxical weakness [31] since cholinesterase antagonists are known to increase muscle twitch tension [32], and are used in reversing the effect of muscle relaxant administered during anesthetics. Stum et al. showed that AChE deficiency in a mouse model might be contributing to muscle stiffness and fatigability [33]. These studies suggest that an AChE deficiency might increase muscle stiffness and fatigue.

Gene-Eden-VIR was designed to target latent viruses in infected individuals. Recently, a clinical study, conducted at the Center for the Biology of Chronic Disease (CBCD), showed that Gene-Eden VIR is antiviral [34]. Since GABP·p300 is a transactivator of several respiratory chain genes, the Microcompetition with Foreign DNA theory predicts that Gene-Eden-VIR will increase the production of cellular energy, and the overall level of energy available to the infected host. Also, since GABP.p300 is a transactivator of several neuromuscular genes, the theory predicts that Gene-Eden-VIR will decrease muscle stiffness and muscle fatigue in the infected host.

This study was designed to test these predictions.

\section{Methods}

\subsection{Ethics Statement}

An informed consent was obtained from all subjects prior to conducting the phone interviews.

\subsection{Objective and Framework}

We used this post marketing study to test the efficacy, safety, and optimal use of Gene-Eden-VIR on individuals suffering from fatigue. Specifically, we tested the effect of Gene-Eden-VIR on the feeling of general, physical, and mental fatigue. The study followed FDA guidelines.

\subsection{Treatment}

Gene-Eden-VIR is a dietary supplement. A capsule of Gene-Eden-VIR includes $100 \mathrm{mg}$ of quercetin, $150 \mathrm{mg}$ of 
green tea extract, $50 \mathrm{mg}$ of cinnamon extract, $25 \mathrm{mg}$ of licorice extract, and $100 \mathrm{mcg}$ of selenium. The treatment included 1, 2, 3, or 4 capsules per day, and lasted 2 to 54 weeks.

\subsection{Questionnaire}

As of today, there is no objective tool to measure fatigue. Currently, researchers and clinician assess fatigue assessments by using a number of subjective scales. We discovered that none of these tools are relevant for our research. Therefore, we used a self-developed questionnaire called the Fatigue Natural Origin Treatment Clinical Questionnaire (F-NotCiq). The F-NotCiq questionnaire is a patient reported outcome (PRO) instrument. We successfully used a version of this tool in our previous studies. The purpose of a PRO instrument is to capture the patient's experience. In our current study, our main endpoint was the feeling of fatigue. Meaning, the objective of the study was to measure the effect of treatment with Gene-Eden-VIR on the feeling of fatigue as reported by the treated participants.

The study collected the answers to the F-NotCiq instrument by phone interviews. We used two independent companies that specialized in outbound call services for performing the interviewers, one company from the US and one from Israel. The interviewers were blinded to the objective of the study. All interviews were recorded.

\subsection{Population}

The study randomly selected participants from the Gene-Eden VIR customer database that includes all GeneEden-VIR current and past customers. The traditional response rate to phone interviews is $10 \%-15 \%$. Since the study was aiming to collect 100 - 150 interviews, the study used a computerized system to randomly create a call list of 1000 customers. The final list of participants consisted of 100 Americans, both male and female, ages 20 to 66, infected with the latent HPV, EBV, HSV, HCMV, and HCV.

Since the objective of the study was to test the effect of Gene-Eden-VIR on the feeling of fatigue, the study excluded participants who reported no feeling of general, physical, or mental fatigue. That is, it excluded participants who reported a 7 point score on the pre-treatment question in these sections. Such score indicates that the participant does not suffer from the specific aspect of fatigue indicated by the question regardless of the treatment the participant actually received. This exclusion still preserved the intention to treat (ITT) principle.

At the start of the study, all participants reported a feeling of fatigue. Specifically, 98 reported a feeling of general fatigue, 90 reported a feeling of physical fatigue, and 79 reported a feeling of mental fatigue.

The study considered participants, who stopped taking Gene-Eden-VIR for a month or more before data collection, as past users. All other participants were considered as present users.

\subsection{Controls}

The Gene-Eden-VIR post marketing study includes a pre-treatment concurrent control, and an historical control. To create an historical control, we divided the original test group into two subgroups, present users and past users. Generally, an historical control is a separate group. However, since we did not have a separate group of nonusers, we used the past users as a proxy for an historical control.

\subsection{Statistical Analysis}

We tested the statistical difference between the score of "pre-treatment", which is the numeric answer each participant used to describe his level of fatigue before the treatment started, to the score of "post-treatment", which is the numeric answer each participant used to describe his level of fatigue after the treatment was completed. We also calculated the delta $(\Delta)$, that is, the difference in scores between the answers to the pre-treatment and post-treatment question. Then, we tested the statistical difference between the deltas. These tests were performed in a then-test model for both present and past users.

Statistical analysis was performed using a two-tail t-test assuming unequal variances.

The research defined the primary endpoint as a statistically significant increase in the score from pre-treatment to post-treatment on the raw answers and on the deltas.

\section{Results}

The participants reported no side effects following treatment with Gene-Eden-VIR. 
Following treatment with Gene-Eden-VIR, 72 out of the 98 participants (73.47\%), who reported a feeling of general fatigue, reported a decrease in their fatigue, 56 out of the 90 (62.22\%), who reported a feeling of physical fatigue, reported a decrease in their fatigue, and 36 out of the 79 (47.36\%), who reported a feeling of mental fatigue, reported a decrease in their fatigue. Moreover, out of the $73(73 \%)$ participants, who reported a feeling of both physical and mental fatigue, 62 (84.93\%), reported a decrease in at least one of those types of fatigue (Table 1).

Following treatment with Gene-Eden-VIR, the participants reported a statistically significant decrease in every aspect of fatigue tested in the study (Table 2).

We could not test for a dose effect since the number of participants who took 1, 3 or 4 capsules per day was too small for statistical analysis.

To test for a duration effect, we compared the change $(\Delta)$ from pre-treatment to post-treatment in participants who took Gene-Eden-VIR for less then two months and those who took Gene-Eden-VIR for two months or more. The results showed that participants who took Gene-Eden-VIR for the longer period reported a larger decrease in their feeling of fatigue. Specifically, the participants reported a statistically significant decrease in their feeling of general fatigue, and in their feeling of both physical and mental fatigue (Table 3).

\begin{tabular}{|c|c|}
\hline Section & $\mathbf{N}^{*}$ \\
\hline \multicolumn{2}{|c|}{ F-General fatigue } \\
\hline Pre-T general fatigue ${ }^{* *}$ & $98 / 100(98.00 \%)$ \\
\hline Post-T decrease in general fatigue ${ }^{* * *}$ & 72/98 (73.47\%) \\
\hline \multicolumn{2}{|c|}{ P-Physical fatigue } \\
\hline Pre-T physical fatigue & $90 / 100(90.00 \%)$ \\
\hline Post-T decrease in physical fatigue & $56 / 90(62.22 \%)$ \\
\hline \multicolumn{2}{|c|}{ M-Mental fatigue } \\
\hline Pre-T mental fatigue & 79/100 (79.00\%) \\
\hline Post-T decrease in mental fatigue & $36 / 79(47.36 \%)$ \\
\hline \multicolumn{2}{|c|}{ Pre-T fatigue } \\
\hline General & $98 / 100(98.00 \%)$ \\
\hline Phys. and/or ment. & $95 / 100(95.00 \%)$ \\
\hline Phys. + gen., or ment.+ gen. & $93 / 100(93.00 \%)$ \\
\hline Phys. + gen. & 89/100 (89.00\%) \\
\hline Ment. + gen. & $77 / 100(77.00 \%)$ \\
\hline Gen. + phys. + ment. & 73/100 (73.00\%) \\
\hline General + no phys. + no ment. & $4 / 100(4.00 \%)$ \\
\hline \multicolumn{2}{|c|}{ Post-T Decrease in: } \\
\hline gen. & 72/98 (73.47\%) \\
\hline phys. and/or ment. & 62/73 (84.93\%) \\
\hline gen. + phys. or gen. + ment. & $54 / 73(73.97 \%)$ \\
\hline gen. + phys. & $51 / 89(57.30 \%)$ \\
\hline gen. + ment. & $34 / 73(46.57 \%)$ \\
\hline phys. and ment. & 32/73 (43.83\%) \\
\hline
\end{tabular}

${ }^{*} \mathrm{~N}$ is number of Participants, ${ }^{* *}$ Pre-T is Pre Treatment, ${ }^{* * *}$ Post-T is Post Treatment. gen. is general, phys. is physical, ment. is mental. 
Table 2. Aspects of fatigue, pre-treatment vs. post-treatment.

\begin{tabular}{|c|c|c|c|c|}
\hline Questions & $\mathbf{N}^{\#}$ & Pre-T & Post-T & p Value \\
\hline \multicolumn{5}{|c|}{ General fatigue } \\
\hline F1-Level of energy & 88 & 4.65 & 5.74 & $<0.001$ \\
\hline F2-Tiredness & 85 & 4.14 & 5.36 & $<0.001$ \\
\hline F3-Ability to sleep & 82 & 4.44 & 5.23 & $<0.001$ \\
\hline \multicolumn{5}{|c|}{ Physical fatigue } \\
\hline P1—Strength & 70 & 4.77 & 5.49 & $<0.001$ \\
\hline P2-Ability to do your job & 43 & 4.95 & 5.51 & 0.05 \\
\hline P3-Ability to exercise & 50 & 4.63 & 5.58 & 0.001 \\
\hline P4_Ability to do housework & 47 & 4.89 & 5.53 & 0.018 \\
\hline P5-Ability to get out of bed & 55 & 4.42 & 5.36 & $<0.001$ \\
\hline P6-Stiffness & 63 & 4.51 & 5.13 & 0.013 \\
\hline \multicolumn{5}{|c|}{ Mental fatigue } \\
\hline M1-Attention span & 68 & 4.85 & 5.46 & 0.003 \\
\hline M2-Ability to form thoughts & 53 & 5.09 & 5.57 & 0.033 \\
\hline M3-Ability to concentrate & 60 & 4.80 & 5.47 & 0.005 \\
\hline M4—Ability to remember & 69 & 4.86 & 5.43 & 0.002 \\
\hline
\end{tabular}

The statistical analysis was conducted using one score per participant. ${ }^{\#} \mathrm{~N}$ is Number of Participants, Pre-T is Pre Treatment, Post-T is Post Treatment.

\section{Table 3. Duration of treatment.}

\begin{tabular}{clll}
\hline Duration of Treatment & $\mathbf{N}^{*}$ & $\boldsymbol{\Delta}^{*}$ & Stat. \\
\hline \multicolumn{4}{c}{ General fatigue } \\
Less than 2 Months & 32 & 0.45 & $\mathrm{P}=0.03, \mathrm{n}=65$ \\
2 Months or More & 33 & 0.84 &
\end{tabular}

Physical or mental fatigue

\begin{tabular}{lccc} 
Less than 2 Months & 34 & 0.44 & $\mathrm{P}=0.05, \mathrm{n}=70$ \\
2 Months or More & 36 & 0.76 & \\
\hline
\end{tabular}

${ }^{*} \Delta$ is change from Pre-T to Post-T. Statistical analysis was performed using the change in scores from Pre-T to Post-T reported by present users only. The analysis used one score per participant equal to the average of the answers to questions F1 F3 for general, and questions P1 - P6 and M1 - M4 for physical and mental.

To test for a possible interviewer bias, we compared the change $(\Delta)$ from pre-treatment to post-treatment collected by the American and the Israeli call centers. The change was tested for both feeling of general fatigue, and for feeling of physical or mental fatigue. In both cases, the difference between the answers was statistically not significant ( $p=0.79, n=254$, for general, and $p=0.49, n=582$, for physical or mental, Table 4). This means that although the centers included different interviewers from different cultures working at different times of day, Americans working during the day and Israelis working during the night, the answers were similar. Hence, the results showed no interviewer bias (Table 4).

To test for a possible selection bias, we compared the change $(\Delta)$ from pre-treatment to post-treatment by the past and present users of Gene-Eden-VIR. The change was tested for both feeling of general fatigue, and for 
feeling of physical or mental fatigue. In both cases, the difference between the answers was statistically insignificant ( $p=0.70, n=247$, for general, $p=0.15, n=568$, for physical or mental, Table 6). This means that, statistically, the answers by the present users are the same as the answers by the past users, and therefore, there was no selection bias (Table 5).

An issue unique to natural products is the concern about the therapeutic consistency of marketed products. Please refer to the discussion on this issue in the FDA guidelines for botanical New Drug Applications (NDA) [35]. To test the therapeutic consistency of Gene-Eden-VIR, we compared the two batches used by the participants. The capsules in these batches were produced at two different manufacturing sites, and completed about 10 months apart. The results showed that the answers given by the participants who used the capsules from Batch 1 were the same as those given by the participants who used the capsules from Batch $2(p=0.62, n=256$, for general, $\mathrm{p}=0.66, \mathrm{n}=574$, for physical or mental, Table 6). Hence, the results indicated that, although GeneEden-VIR is a natural product, its formula has therapeutic consistency (Table 6).

\section{Discussion}

This post marketing clinical study showed that treatment with Gene-Eden-VIR safely decreased the feeling of

Table 4. USA vs. Israel call centers.

\begin{tabular}{|c|c|c|c|c|c|}
\hline & \multicolumn{2}{|c|}{ USA } & \multicolumn{2}{|c|}{ Israel } & \multirow{2}{*}{ Stat. } \\
\hline & $\mathbf{N}$ & Score & $\mathbf{N}$ & Score & \\
\hline \multicolumn{6}{|c|}{ General fatigue $^{*}$} \\
\hline$\Delta$ & 153 & 1.03 & 101 & 1.07 & $\mathrm{p}=0.79 \mathrm{n}=254$ \\
\hline \multicolumn{6}{|c|}{ Physical or mental ${ }^{* *}$} \\
\hline$\Delta$ & 323 & 0.69 & 259 & 0.63 & $\mathrm{p}=0.49 \mathrm{n}=582$ \\
\hline
\end{tabular}
up to ten answers per participant.

Table 5. Past vs. present users.

\begin{tabular}{|c|c|c|c|c|c|}
\hline & \multicolumn{2}{|c|}{ Present } & \multicolumn{2}{|c|}{ Past } & \multirow{2}{*}{ Stat. } \\
\hline & $\mathbf{N}$ & Score & $\mathbf{N}$ & Score & \\
\hline \multicolumn{6}{|c|}{ General fatigue } \\
\hline$\Delta$ & 181 & 1.06 & 68 & 0.99 & $p=0.70 n=247$ \\
\hline \multicolumn{6}{|c|}{ Physical or mental fatigue ${ }^{* *}$} \\
\hline$\Delta$ & 411 & 0.61 & 157 & 0.77 & $p=0.15 n=568$ \\
\hline
\end{tabular}

*Data may include up to three answers per participant. ${ }^{* *}$ Data may include up to ten answers per participant.

Table 6. Batch 1 vs. Batch 2.

\begin{tabular}{|c|c|c|c|c|c|}
\hline & \multicolumn{2}{|c|}{ Batch 1} & \multicolumn{2}{|c|}{ Batch 2} & \multirow{2}{*}{ Stat. } \\
\hline & $\mathbf{N}$ & Score & $\mathbf{N}$ & Score & \\
\hline \multicolumn{6}{|c|}{ General fatigue } \\
\hline$\Delta$ & 147 & 1.06 & 109 & 0.98 & $p=0.62 n=256$ \\
\hline \multicolumn{6}{|c|}{ Physical or mental fatigue ${ }^{* *}$} \\
\hline$\Delta$ & 318 & 0.66 & 256 & 0.63 & $\mathrm{p}=0.66 \mathrm{n}=574$ \\
\hline
\end{tabular}

*Data may include up to three answers per participant. ${ }^{* *}$ Data may include up to ten answers per participant. 
fatigue in individuals infected with a latent virus. These results support the Microcompetition with Foreign DNA theory.

The results are consistent. We observed a statistically significant decrease in the feeling of general, physical, and mental fatigue.

The results also showed a duration effect. Participants treated for two months or more reported a larger decrease in their feeling of fatigue compared to those treated for less then two months.

The results are robust. They showed no interviewer bias, no selection bias, and therapeutic consistency of the Gene-Eden-VIR formula under varying manufacturing conditions.

The results in our study are consistent with the results reported in another study that tested the effect of an antiviral drug on fatigue. In this study, Watt et al. 2012 showed that treatment with the antiviral drug Valganciclovir improved physical and cognitive performance in patients suffering from fatigue. The study also showed that longer treatments were correlated with better outcomes [36].

This post marketing clinical study does not include a placebo control, that is, it is not a double-blinded study. Placebo controlled studies are the gold standard in medical research in pre-marketing clinical studies. However, except in rare cases, post marketing studies do not use placebo controls. They use other controls recommended by the FDA.

The FDA guidance lists five types of controls for both pre-marketing and post marketing studies: 1) Placebo Concurrent Control, 2) Pre-treatment Concurrent Control, 3) Dose-response Concurrent Control, 4) Active (Positive) Concurrent Control, 5) External Control (Including Historical Control). The External Control "can be a group of patients treated at an earlier time (historical control)" [37].

The Gene-Eden-VIR post marketing study is a change from baseline study that includes a pre-treatment concurrent control and a proxy for an historical control.

The results are not likely to be a placebo effect. The current predominant and well proven theories on the placebo effect suggest that its main mechanisms are conditioned reflexes and patient expectations [38]. The GeneEden-VIR product literature does not mention the possibility of a change in the feeling of fatigue. Hence, the participants in this study could not have been primed for, or expect the reported effects. This lack of conditioned reflexes and patient expectations minimizes the possibility of a placebo effect, and supports the possibility of a physiological effect.

All participants who started the study completed it; therefore, the study has no follow-up bias. It should be noted that although we tested for some biases, others are still possible, for instance, the non-responsive bias.

This study relies on patient reported outcomes (PROs). Past studies showed that PROs had a significant role in the development and evaluation of new medicines [39]. According to the FDA, PROs are a valid and valuable source for measuring the efficacy of new drugs. They are reliable enough to warrant an approval of a label claim for a new drug. From the years 1997 to 2002, the FDA approved 23 new drugs based on results obtained in studies that used only PRO endpoints. They include six anti-migraine products (Amerge ${ }^{\circledR}$, Ax-ert $^{\circledR}$ ), several anti-epileptics $\left(\right.$ Gabitril $^{\circledR}$, Keppra $^{\circledR}$ ), and a variety of other therapy classes (Tamiflu ${ }^{\circledR}$, Relenza ${ }^{\circledR}$ ).

The FDA regards PROs as a valid and valuable source of data. The scientific community also believes that PROs are valid and useful. Many major journals published clinical studies that use patient reported outcomes. The trust of the FDA and the scientific community in PROs should convince the medical community, and specifically, doctors, to trust studies that use PROs when evaluating the benefits of new treatments.

A possible criticism of the F-NotCiq is that it uses a subjective scale, and therefore, may be producing biased results. However, the feeling of fatigue is essentially a subjective experience, and therefore, cannot be measured objectively.

The size of the study group is a major concern in clinical studies. A small group may fail to show a positive effect. In addition, a small group might misrepresent the diversity in the population. The standard principle for multivariate behavioral research is at least 10 patients for one endpoint, that is, for one dependent variable [40]. This study included one endpoint, the change in the feeling of fatigue from pre-treatment to post-treatment. The population included 100 individuals. Hence, the size of the study group is adequate.

One might also question the reliability of the participants recall due to the long duration of the period under investigation (up to 54 weeks). This study used a "then-test" method. This method, which is also known as the retrospective pre-test-post-test design method, asks participants at the post-test period to think back to the pre-test period, and retrospectively rate their condition. The "response shift" is defined as the difference between the "pre-test" and the "then-test" ratings. Currently, the response shift is a well documented and extensively re- 
searched phenomenon [41]. According to the literature on "response shifts", participants may alter their internal standards, values, or conceptualization of their quality of life when experiencing changes in health states. The response shift can affect or distort the reported scores and undermine the credibility of the observed medical or psychosocial effects. Many studies reported that, after participants experience an improvement in their health, a then-test tends to show a decrease in the initial assessment of the original level of well being.

Since this clinical study uses the "then-test" method, we tested for a possible response shift by comparing the answers to the pre-treatment question at less than two months and at two months or more. The results showed a statistically significant increase in the pre-treatment score over time. The results indicated that the participants experienced a response shift, however, in the opposite direction from what was expected. This response shift suggests that the participants do not tend to exaggerate, but tend to forget how bad their level of fatigue was before taking Gene-Eden-VIR. The tendency to forget adds support to the statistical significance of the results in this study.

Latent infections are very common. The worldwide rates of HSV infection range from $65 \%$ to $90 \%$ [42]. The rate of EBV infection is about $95 \%$ of the world's adult population. Epidemiological studies reported that the rate of HPV infection, over the lifetime of people in the USA between the ages of 15 and 50, is about 75\% [43]. The rate of CMV infection in individuals in the USA at the age of 6 and older is 58.9\%, and in certain countries it reaches $100 \%$ [44]. In most infected individuals, an efficient immune system controls the number of latent viruses [45]. However, when the environment changes, such as during stress, injury, aging, while taking certain medications, etc., the immune system weakens. As a result, Microcompetition with the higher number of latent viral particles overwhelms the cellular genetic system. One result of this intense Microcompetition is a feeling of fatigue.

\section{Conclusion}

This post marketing clinical study showed that treatment with Gene-Eden-VIR safely decreased the feeling of general, physical, and mental fatigue in individuals infected with a latent virus. Since most individuals are infected with a latent virus, health care practitioners should recommend Gene-Eden-VIR as a first line treatment for fatigue. The results of this post marketing clinical study support the Microcompetition with Foreign DNA theory.

\section{References}

[1] Chalder, T., Neeleman, J., Reme, S.E., Power, M. and Wessely, S. (2010) Factors Associated with Acute Fatigue in Primary Care. Psychological Medicine, 40, 1289-1295. http://dx.doi.org/10.1017/S0033291709992327

[2] Chaudhuri, A. and Behan, P.O. (2004) Fatigue in Neurological Disorders. Lancet, 363, 978-988. http://dx.doi.org/10.1016/S0140-6736(04)15794-2

[3] Shen, J., Barbera, J. and Shapiro, C.M. (2006) Distinguishing Sleepiness and Fatigue: Focus on Definition and Measurement. Sleep Medicine Reviews, 10, 63-76. http://dx.doi.org/10.1016/j.smrv.2005.05.004

[4] Pantry, S.N., Medveczky, M.M., Arbuckle, J.H., Luka, J., Montoya, J.G., Hu, J., Renne, R., Peterson, D., Pritchett, J.C., Ablashi, D.V. and Medveczky, P.G. (2013) Persistent Human Herpesvirus-6 Infection in Patients with an Inherited Form of the Virus. Journal of Medical Virology, 85, 1940-1946. http://dx.doi.org/10.1002/jmv.23685

[5] Karaivazoglou, K., Iconomou, G., Triantos, C., Hyphantis, T., Thomopoulos, K., Lagadinou, M., Gogos, C., Labropoulou-Karatza, C. and Assimakopoulos, K. (2010) Fatigue and Depressive Symptoms Associated with Chronic Viral Hepatitis Patients. Health-Related Quality of Life (HRQOL). Annals of Hepatology, 9, 419-427.

[6] Barroso, J., Hammill, B.G., Leserman, J., Salahuddin, N., Harmon, J.L. and Pence, B.W. (2010) Physiological and Psychosocial Factors that Predict HIV-Related Fatigue. AIDS and Behavior, 14, 1415-1427. http://dx.doi.org/10.1007/s10461-010-9691-2

[7] Kondo, K. (2006) Post-Infectious Fatigue. Japan Medical Association Journal, 49, 27-33.

[8] U.S. Food and Drug Administration (FDA) (2001) Long term Follow-Up Of Gene Transfer Patients. 31st Biological Response Modifiers Advisory Committee Meeting Gaithersburg, Maryland. http://www.fda.gov/ohrms/dockets/ac/01/briefing/3794b1_01.htm

[9] Polansky, H. (2003) Microcompetition with Foreign DNA and the Origin of Chronic Disease. Center for the Biology of Chronic Disease (CBCD) Publishing.

[10] Liu, B.H., Wang, X., Ma, Y.X. and Wang, S. (2004) CMV Enhancer/Human PDGF-Beta Promoter for Neuron-Spe- 
cific Transgene Expression. Gene Therapy, 11, 52-60. http://dx.doi.org/10.1038/sj.gt.3302126

[11] Slobedman, B. and Mocarski, E.S. (1999) Quantitative Analysis of Latent Human Cytomegalovirus. Journal of Virology, 73, 4806-4812.

[12] Adam, G.I., Miller, S.J., Ulleras, E. and Franklin, G.C. (1996) Cell-Type-Specific Modulation of PDGF-B Regulatory Elements via Viral Enhancer Competition: A Caveat for the Use of Reference Plasmids in Transient Transfection Assays. Gene, 178, 25-29. http://dx.doi.org/10.1016/0378-1119(96)00318-6

[13] Scarpulla, R.C. (1997) Nuclear Control of Respiratory Chain Expression in Mammalian Cells. Journal of Bioenergetics and Biomembranes, 29, 109-119. http://dx.doi.org/10.1023/A:1022681828846

[14] Scarpulla, R.C. (1999) Nuclear Transcription Factors in Cytochrome c and Cytochrome Oxidase Expression. In: Papa, S., Guerrieri, F. and Tager, J.M., Eds., Frontiers of Cellular Bioenergetics: Molecular Biology, Biochemistry, Physiopathology, Plenum, New York, 553-591. http://dx.doi.org/10.1007/978-1-4615-4843-0_22

[15] Ongwijitwat, S. and Wong-Riley, M.T. (2005) Is nuclear Respiratory Factor 2 a Master Transcriptional Coordinator for All Ten Nuclear-Encoded Cytochrome c Oxidase Subunits in Neurons? Gene, 360, 65-77. http://dx.doi.org/10.1016/j.gene.2005.06.015

[16] Ongwijitwat, S., Liang, H.L., Graboyes, E.M. and Wong-Riley, M.T. (2006) Nuclear Respiratory Factor 2 Senses Changing Cellular Energy Demands and Its Silencing Down-Regulates Cytochrome Oxidase and Other Target Gene mRNAs. Gene, 374, 39-49. http://dx.doi.org/10.1016/j.gene.2006.01.009

[17] Larsson, N.G., Wang, J.M., Wilhelmsson, H., Oldfors, A., Rustin, P., Lewandoski, M., Barsh, G.S. and Clayton, D.A. (1998) Mitochondrial Transcription Factor A Is Necessary for mtDNA Maintenance and Embryogenesis in Mice. Nature Genetics, 18, 231-236. http://dx.doi.org/10.1038/ng0398-231

[18] Rantanen, A., Jansson, M., Oldfors, A. and Larsson, N.G. (2001) Downregulation of Tfam and mtDNA Copy Number during Mammalian Spermatogenesis. Mammalian Genome, 12, 787-792. http://dx.doi.org/10.1007/s00335-001-2052-8

[19] McCulloch, V., Seidel-Rogol, B.L. and Shadel, G.S. (2002) A Human Mitochondrial Transcription Factor Is Related to RNA Adenine Methyltransferases and Binds S-Adenosylmethionine. Molecular and Cellular Biology, 22, 1116-1125. http://dx.doi.org/10.1128/MCB.22.4.1116-1125.2002

[20] Falkenberg, M., Gaspari, M., Rantanen, A., Trifunovic, A., Larsson, N.G. and Gustafsson, C.M. (2002) Mitochondrial Transcription Factors B1 and B2 Activate Transcription of Human mtDNA. Nature Genetics, 31, 289-294. http://dx.doi.org/10.1038/ng909

[21] Au, H.C. and Scheffler, I.E. (1998) Promoter Analysis of the Human Succinate Dehydrogenase Iron-Protein Gene. Both Nuclear Respiratory Factors NRF-1 and NRF-2 Are Required. European Journal of Biochemistry, 251, 164-174. http://dx.doi.org/10.1046/j.1432-1327.1998.2510164.x

[22] Elbehti-Green, A., Au, H.C., Mascarello, J.T., Ream-Robinson, D. and Scheffler, I.E. (1998) Characterization of the Human SDHC Gene Encoding One of the Integral Membrane Proteins of Succinate-Quinone Oxidoreductase in Mitochondria. Gene, 213, 133-140. http://dx.doi.org/10.1016/S0378-1119(98)00186-3

[23] Hirawake, H., Taniwaki, M., Tamura, A., Amino, H., Tomitsuka, E. and Kita, K. (1999) Characterization of the Human SDHD Gene Encoding the Small Subunit of Cytochrome b (cybS) in Mitochondrial Succinate-Ubiquinone Oxidoreductase. Biochimica et Biophysica Acta (BBA)—Bioenergetics, 1412, 295-300. http://dx.doi.org/10.1016/S0005-2728(99)00071-7

[24] Khurana, T.S., Rosmarin, A.G., Shang, J., Krag, T.O., Das, S. and Gammeltoft, S. (1999) Activation of Utrophin Promoter by Heregulin via the ets-Related Transcription Factor Complex GA-Binding Protein Alpha/Beta. Molecular and Cellular Biology, 10, 2075-2086. http://dx.doi.org/10.1091/mbc.10.6.2075

[25] Chan, R.Y., Boudreau-Larivière, C., Angus, L.M., Mankal, F.A. and Jasmin, B.J. (1999) An Intronic Enhancer Containing an N-Box Motif Is Required for Synapse- and Tissue-Specific Expression of the Acetylcholinesterase Gene in Skeletal Muscle Fibers. Proceedings of the National Academy of Sciences, 96, 4627-4632. http://dx.doi.org/10.1073/pnas.96.8.4627

[26] O’Leary, D.A., Noakes, P.G., Lavidis, N.A., Kola, I., Hertzog, P.J. and Ristevski, S. (2007) Targeting of the ETS Factor Gabp Alpha Disrupts Neuromuscular Junction Synaptic Function. Molecular and Cellular Biology, 27, 3470-3480. http://dx.doi.org/10.1128/MCB.00659-06

[27] Tinsley, J.M., Fairclough, R.J., Storer, R., Wilkes, F.J., Potter, A.C., Squire, S.E., Powell, D.S., Cozzoli, A., Capogrosso, R.F., Lambert, A., Wilson, F.X., Wren, S.P., De Luca, A. and Davies, K.E. (2011) Daily Treatment with SMTC1100, a Novel Small Molecule Utrophin Upregulator, Dramatically Reduces the Dystrophic Symptoms in the mdx Mouse. PLoS One, 6, e19189 http://www.plosone.org/article/info\%3Adoi\%2F10.1371\%2Fjournal.pone.0019189 http://dx.doi.org/10.1371/journal.pone.0019189

[28] Faber, C.G., Molenaar, P.C., Vles, J.S., Bonifati, D.M., Verschuuren, J.J., van Doorn, P.A., Kuks, J.B., Wokke, J.H., Beeson, D. and De Baets, M. (2009) AChR Deficiency Due to Epsilon-Subunit Mutations: Two Common Mutations in 
the Netherlands. Journal of Neurology, 256, 1719-1723. http://dx.doi.org/10.1007/s00415-009-5190-7

[29] Sieb, J.P., Kraner, S., Rauch, M. and Steinlein, O.K. (2000) Immature End-Plates and Utrophin Deficiency in Congenital Myasthenic Syndrome Caused by Epsilon-AChR Subunit Truncating Mutations. Human Genetics, 107, 160-164. http://dx.doi.org/10.1007/s004390000359

[30] Payne, J.P., Hughes, R. and Al Azawi, S. (1980) Neuromuscular Blockade by Neostigmine in Anaesthetized Man. British Journal of Anaesthesia, 52, 69-76. http://dx.doi.org/10.1093/bja/52.1.69

[31] Caldwell, J.E. (2009) Clinical Limitations of Acetylcholinesterase Antagonists. Journal of Critical Care, 24, 21-28. http://dx.doi.org/10.1016/j.jcrc.2008.08.003

[32] Barber, H.E., Calvey, T.N. and Muir, K.T. (1979) The Relationship between the Pharmacokinetics, Cholinesterase Inhibition and Facilitation of Twitch Tension of the Quaternary Ammonium Anticholinesterase Drugs, Neostigmine, Pyridostigmine, Edrophonium and 3-Hydroxyphenyltrimethylammonium. British Journal of Pharmacology, 66, 525530. http://dx.doi.org/10.1111/j.1476-5381.1979.tb13690.x

[33] Stum, M., Girard, E., Bangratz, M., Bernard, V., Herbin, M., Vignaud, A., Ferry, A., Davoine, C.S., Echaniz-Laguna, A., René, F., Marcel, C., Molgó, J., Fontaine, B., Krejci, E. and Nicole, S. (2008) Evidence of a Dosage Effect and a Physiological Endplate Acetylcholinesterase Deficiency in the First Mouse Models Mimicking Schwartz-Jampel Syndrome Neuromyotonia. Human Molecular Genetics, 17, 3166-3179. http://dx.doi.org/10.1093/hmg/ddn213

[34] Polansky, H. and Itzkovitz, E. (2013) Gene-Eden-VIR Is Antiviral: Results of a Post Marketing Clinical Study. Pharmacology \& Pharmacy, 4, 1-8. http://dx.doi.org/10.4236/pp.2013.46A001

[35] US Department of Health and Human Services, Food and Drug Administration, Center for Drug Evaluation and Research (CDER) (2004) Guidance for Industry. Botanical Drug Products. http://www.fda.gov/downloads/Drugs/GuidanceComplianceRegulatoryInformation/Guidances/ucm070491.pdf http://dx.doi.org/10.1002/jmv.23411

[36] Watt, T., Oberfoell, S., Balise, R., Lunn, M.R., Kar, A.K., Merrihew, L., Bhangoo, M.S. and Montoya, J.G. (2012) Response to Valganciclovir in Chronic Fatigue Syndrome Patients with Human Herpesvirus 6 and Epstein-Barr Virus IgG Antibody Titers. Journal of Medical Virology, 84, 1967-1974.

[37] US Department of Health and Human Services, Food and Drug Administration, Center for Drug Evaluation and Research (CDER) (2001) Guidance for Industry, E 10 Choice of Control Group and Related Issues in Clinical Trials. http://www.fda.gov/downloads/RegulatoryInformation/Guidances/ucm129460.pdf

[38] Breidert, M. and Hofbauer, K. (2009) Placebo: Misunderstandings and Prejudices. Deutsches Ärzteblatt International, 106, 751-755.

[39] Willke, R.J., Burke, L.B. and Erickson, P. (2004) Measuring Treatment Impact: A Review of Patient-Reported Outcomes and Other Efficacy Endpoints in Approved Product Labels. Controlled Clinical Trials, 25, 535-552. http://dx.doi.org/10.1016/j.cct.2004.09.003

[40] Harvey, P.D. and Keefe, R.S. (2001) Studies of Cognitive Change in Patients with Schizophrenia Following Novel Antipsychotic Treatment. The American Journal of Psychiatry, 158, 176-184. http://dx.doi.org/10.1176/appi.ajp.158.2.176

[41] Schwartz, C.E., Bode, R. and Repucci, N. (2006) The Clinical Significance of Adaptation to Changing Health: A MetaAnalysis of Response Shift. Quality of Life Research, 15, 1533-1550. http://dx.doi.org/10.1007/s11136-006-0025-9

[42] Chayavichitsilp, P., Buckwalter, J.V., Krakowski, A.C. and Friedlander, S.F. (2009) Herpes Simplex. Pediatrics in Review, 30, 119-129.

[43] World Health Organization (WHO) (2010) Viral Cancers. http://www.who.int/vaccine_research/diseases/viral_cancers/en/index1.html

[44] Staras, S.A., S Dollard, C., Radford, K.W., Flanders, W.D., Pass, R.F. and Cannon, M.J. (2006) Seroprevalence of Cytomegalovirus Infection in the United States, 1988-1994. Clinical Infectious Diseases, 43, 1143-1151. http://dx.doi.org/10.1086/508173

[45] Grinde, B. (2013) Herpesviruses: Latency and Reactivation-Viral Strategies and Host Response. Journal of Oral Microbiology, 5, 22766. http://dx.doi.org/10.3402/jom.v5i0.22766 